Trauma Berufskrankh 2016 18 (Suppl 5):S474-S478 DOI 10.1007/s10039-016-0163-4

Online publiziert: 6. Juni 2016

๑) Springer-Verlag Berlin Heidelberg 2016

CrossMark
C. Ries · K. Wegmann · M. Hackl - T. Leschinger · L. P. Müller

Klinik und Poliklinik für Orthopädie und Unfallchirurgie, Universitätsklinik Köln, Köln, Deutschland

\title{
Ellenbogenluxationsfrakturen und Monteggia-Verletzung
}

\section{Posttraumatische Korrekturen und Revisionen}

Der Ellenbogen ist ein komplexes Gelenk, das seine Stabilität neben der knöchernen Führung durch die muskuloligamentären Strukturen erhält. Schwere Verletzungen, welche die Gelenkintegrität und -stabilität kompromittieren, erfordern komplexe Therapiestrategien, wobei das Risiko für postoperative Komplikationen nicht unerheblich ist. Zur Vermeidung eben solcher Komplikationen müssen die anatomischen Strukturen und ihre Bedeutung für die Gelenkbiomechanik berücksichtigt werden.

Im vorliegenden Beitrag werden wichtige Aspekte erläutert, die im Revisionsfall bedacht werden sollten, um das postoperative Ergebnis möglichst positiv zu beeinflussen.

\section{Vollständige Anamnese- erhebung und zielführende Bildgebung}

Bei anstehenden posttraumatischen Korrekturen und Revisionen ist die detaillierte Anamneseerhebung essenziell. Insbesondere vorausgegangene Operationen mit ggf. stattgehabter Transposition des N. ulnaris sollten erfragt oder anhand alter Operationsberichte nachvollzogen werden. Die klinische Untersuchung unter Einschluss der neurovaskulären Strukturen ist ebenso bedeutsam. Bei Hinweisen auf eine neurogene Einschränkung ist die präoperative neurologische Abklärung mittels Elektromyographie/Nervenleitgeschwindigkeit (EMG/NLG) obligat.

Als bildgebendes Verfahren dient zunächst die standardisierte konventionel- le Röntgenaufnahme des Ellenbogengelenkes in 2 Ebenen (a.-p. und streng seitlich). Die sonographische Untersuchung kann zur Detektion einer Seitenbandinstabilität additiv verwendet werden. Ein Vorteil der Sonographie liegt in der dynamischen Untersuchungsmethode, wobei die Untersucherabhängigkeit als Negativkriterium zu erwähnen ist. Das Standarddiagnostikum zur Evaluation einer Bandläsion bleibt die Magnetresonanztomographie (MRT). Zur Beurteilung der knöchernen Strukturen bzw. zum besseren Verständnis der ehemaligen Frakturmorphologie und/oder zur Beurteilung der knöchernen Konsolidierung (Pseudarthrose?) dient die Computertomographie (3-D-Rekonstruktion mit koronarer und sagittaler Schichtung).

Nach vollständiger Diagnostik ist unter Berücksichtigung der initialen Verletzung und der vorliegenden Komplikationen das individuelle Therapieregime festzulegen.
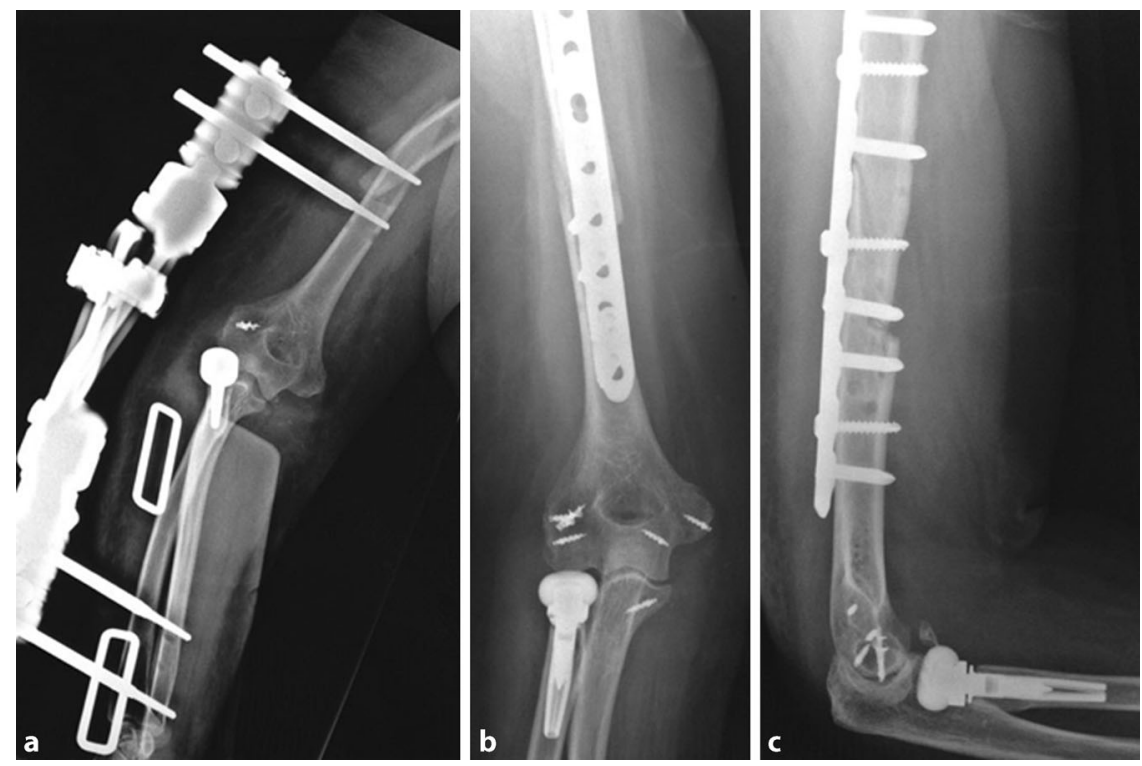

Abb. $1 \Delta$ a Humerusschaftfraktur aufHöhe der radialen Schanz-Schrauben mit begleitender Radialisaffektion bei extern versorgter Terrible-triad-Verletzung mittels Radiuskopfprothese, lateraler Kollateralbandrefixation und Bewegungsfixateur. b, c Revision mit offener Reposition und Plattenosteosynthese der Humerusschaftfraktur. Bei einem Overstuffing der initial eingebrachten Radiuskopfprothese erfolgten der Wechsel und die Neuausrichtung des Implantates (MoPyC Radiuskopf-Prothese, Fa. Tornier). Nach lateraler und medialer Bandrefixation zeigten sich intraoperativ stabile Gelenkverhältnisse, sodass auf einen Fixateur verzichtet werden konnte. (৫ Mit freundl. Genehmigung Prof. Dr. L. P. Müller, all rights reserved) 


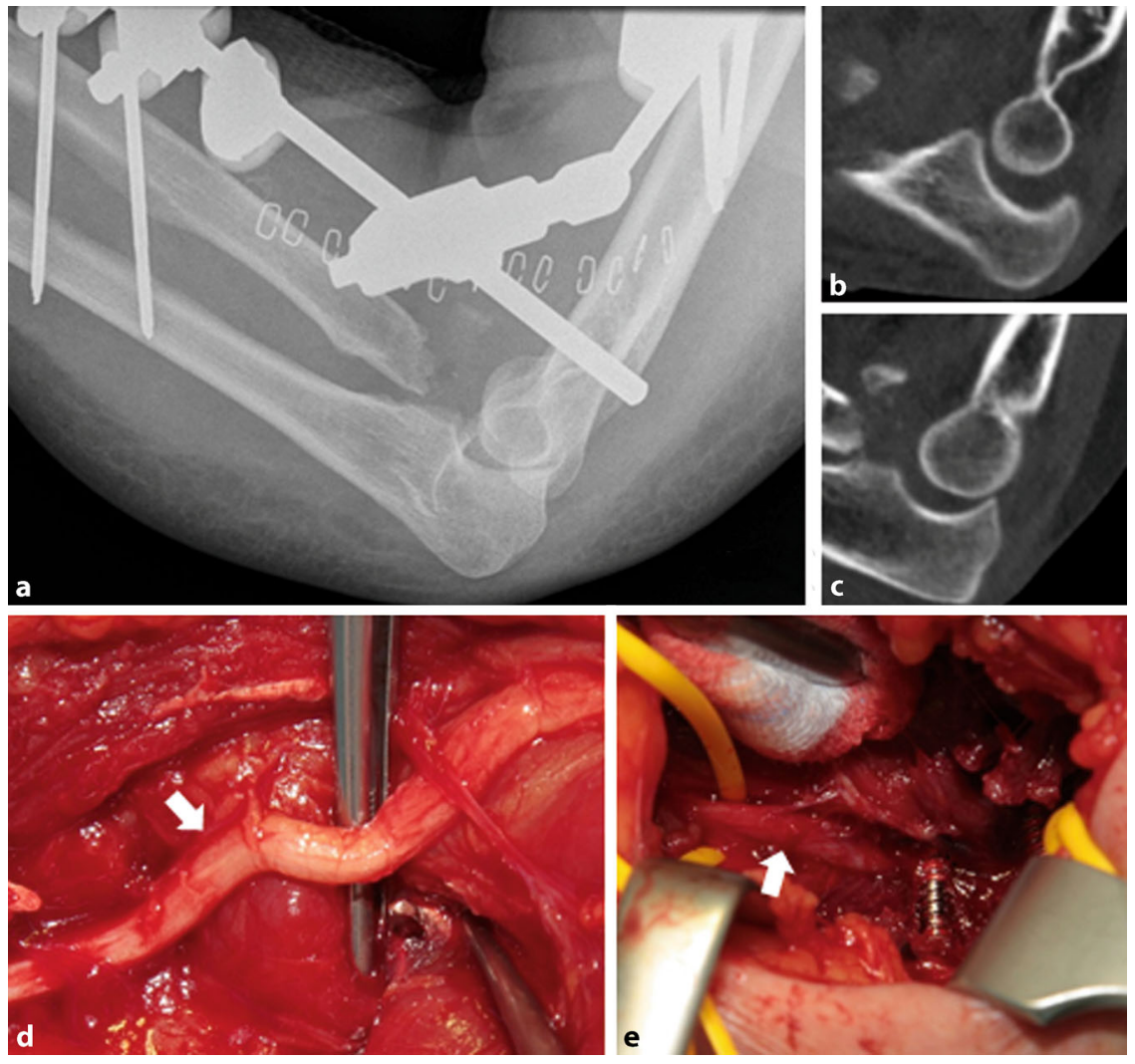

Abb. $2 \Delta$ a Postoperatives Röntgenbild nach extern versorgter Terrible-triad-Verletzung mittels Radiuskopfresektion und Anlage eines gelenküberschreitenden Fixateur externe mit postoperativ bestehender Affektion des N. ulnaris und N. radialis. b, c Humeroulnare Subluxationsstellung in der postoperativen Computertomographie. $\mathbf{d}$ Darstellung des N. ulnaris (Pfeil) im Rahmen des Revisionseingriffes in unmittelbarer Nähe zu den Schanz-Schrauben des Fixateurs. e Der N. radialis (Pfeil) wird durch die eingebrachte Schanz-Schraube unmittelbar kompromittiert. (@ Mit freundl. Genehmigung Prof. Dr. L. P. Müller, all rights reserved)

\section{Terrible-triad-Verletzung}

Die posteriore oder posterolaterale Ellenbogenluxation in Kombination mit einer Fraktur des Radiuskopfes und des Processus coronoideus wird als Terrible-triadVerletzung bezeichnet [1-3]. Hieraus resultiert eine komplexe Ellenbogeninstabilität, die mit einem erhöhten Risiko für eine posttraumatische Bewegungseinschränkung sowie frühzeitige degenerative Gelenkveränderungen mit konsekutiver funktionaler Einschränkung assoziiert ist $[1,2,4,5]$. In der Literatur lässt sich dies durch eine hohe Komplikationsund Revisionsrate belegen [6].

Das übergeordnete Ziel der Therapie ist die Wiederherstellung der Gelenkstabilität durch Rekonstruktion der primären und sekundären Stabilisatoren [3]. Zur Vermeidung von Komplikationen spielt der Erhalt der radialen Säule eine entscheidende Rolle. Die axial auf das Handgelenk einwirkenden Kräfte werden am gestreckten Ellenbogengelenk mit bis zu $60 \%$ über die radiale Säule übertragen [7]. Zudem wirkt der Radiuskopf als wichtiger sekundärer Valgusstabilisator [8]. Bei Wegfall der radialen Säule ist daher - durch die vermehrte humeroulnare Belastung - das Risiko für eine frühzeitige Degeneration in Kombination mit einer relevanten Valgusinstabilität erhöht. Durch neuere winkelstabile, anatomisch präformierte Plattendesigns können zum Teil auch Radiuskopftrümmerfrakturen suffizient adressiert werden. Ist der Rekonstruktionsversuch frustran, sollte der Radiuskopf durch eine Endoprothese ersetzt werden, wobei ein Overstuffing der Prothese mit konsekutiver Arrosion des Capitulums strikt vermieden werden sollte ( $\bullet$ Abb. $1 \mathbf{a}-\mathbf{c})$. Die Ausrichtung der Prothese erfolgt in Bezug auf die laterale Kante des Processus coronoideus, sodass sich die Gelenk- fläche des Radiuskopfersatzes auf Höhe oder bis maximal $0,9 \mathrm{~mm}$ proximal der lateralen Koronoidkante befindet [9]. Die alleinige Radiuskopfresektion sollte nach Möglichkeit nicht erfolgen.

Die Wiederherstellung der Bandstabilität durch Refixation des lateralen Kollateralbandkomplexes ist essenziell. Sollte sich unter intraoperativer Durchleuchtung im Anschluss weiterhin eine Instabilität nachweisen lassen, ist auch das mediale Kollateralband zu refixieren. Im eigenen Vorgehen erfolgt die Bandrefixation - unter Berücksichtigung der knöchernen humeralen Ursprünge - unter Verwendung von Fadenankern. Bei dennoch persistierender Instabilität unter Durchleuchtung trotz erfolgter medialer und lateraler Bandrefixation bzw. Bandrekonstruktion („internal bracing“) empfehlen wir zusätzlich die Anlage eines Bewegungsfixateurs für 6 Wochen, um die Gelenkstabilität positiv zu beeinflussen (- Abb. 2a-e und 3a, b). Bei der Fixateuranlage müssen die Verläufe der neurovaskulären Strukturen bedacht werden. Zur Vermeidung von Nervenläsionen werden die Schanz-Schrauben im eigenen Vorgehen in Mini-open-Technik unter Sicht platziert.

Der Processus coronoideus wirkt insbesondere in Streckstellung Varuskräften entgegen [10] und ist für die Aufrechterhaltung der posterioren Gelenkstabilität ebenso von Bedeutung. Transversale Koronoidfragmente, die über $50 \%$ des Processus einschließen, sollten anatomisch reponiert und fixiert werden [10]. Berücksichtigt werden sollte zudem die anatomische Insertion des anterioren Bündels des medialen Kollateralbandes am Tuberculum subliminus. Bezieht der Frakturverlauf innerhalb des Processus coronoideus die anteriomediale Facette mit ein, so resultiert eine mediale Instabilität [11], die in der operativen Therapie adressiert werden muss, um die Valgusstabilität wiederherzustellen.

\section{Radiuskopfluxationsfrakturen (Mason Typ IV)}

Kommt es zu einer hochenergetischen axialen Belastung des pronierten Unterarmes - wie bei einem abgefangenen Sturz -, werden die osteoligamentären 
Strukturen stark belastet, sodass das Risiko für eine Radiuskopffraktur und auch das Risiko für eine ligamentäre Verletzung steigen [12]. Bereits einfache $\mathrm{Ra}$ diuskopffrakturen können mit ligamentären Begleitverletzungen assoziiert sein [13]. Die Schwere der Radiuskopffraktur korreliert mit dem Verletzungsrisiko der Membrana interossea mit konsekutiver Beeinträchtigung der longitudinalen Unterarmstabilität [14]. Die klinische Untersuchung der Bandstabilität unter Einschluss des distalen Radioulnargelenkes ist daher essenziell. Bei Verdacht auf eine Beteiligung der Membrana interossea sollte schon frühzeitig eine MRT-Diagnostik des Unterarmes erfolgen, um eine Verletzung der Membran im Sinne einer Essex-Lopresti-Läsion auszuschließen.

Die alleinige Resektion des Radiuskopfes führt zur humeroulnaren Überbelastung mit frühzeitiger Degeneration und begünstigt eine Valgusinstabilität. Zudem kann es durch die fortwährende Belastung der Membrana interossea zu einer longitudinalen Instabilität (chronische Essex-Lopresti-Läsion) kommen, sodass sekundär eine radiale Verkürzung mit konsekutivem ulnarem Vorschub resultiert [15, 16]. Der Erhalt der radialen Säule durch Rekonstruktion bzw. Ersatz des Radiuskopfes ist bei Radiuskopfluxationsfrakturen ebenso wichtig wie im Falle der Terrible-triad-Verletzung des Ellenbogens. Das operative Weichteilmanagement - mit Rekonstruktion der Kollateralbandkomplexe und im Falle der chronischen Essex-Lopresti-Läsion ggf. auch durch Rekonstruktion der Membrana interossea - ist für das funktionale Ergebnis wichtig.

\section{Monteggia-Verletzung}

Die von G. B. Monteggia [17] erstmals 1814 beschriebene Kombinationsverletzung aus proximaler Ulnafraktur mit begleitender Radiuskopfluxation stellt ebenso eine schwerwiegende Verletzung des Ellenbogens dar, die mit hohen Komplikations- und Revisionsraten einhergeht $[18,19]$. Ist der Radiuskopf nicht nur luxiert, sondern zusätzlich frakturiert, spricht man von einer „Monteggialike-lesion“. Der Erhalt der radialen Säule

Trauma Berufskrankh 2016 · 18 (Suppl 5):S474-S478 DOI 10.1007/s10039-016-0163-4

(C) Springer-Verlag Berlin Heidelberg 2016

C. Ries · K. Wegmann · M. Hackl · T. Leschinger · L. P. Müller

Ellenbogenluxationsfrakturen und Monteggia-Verletzung.
Posttraumatische Korrekturen und Revisionen

\section{Zusammenfassung}

Im Falle einer Revision bzw. von posttraumatischen Komplikationen ist das weitere therapeutische Vorgehen - aufgrund der anatomisch-biomechanischen Besonderheiten des Ellenbogengelenkes - oftmals kompliziert und erfordert individuelle Entscheidungen. Die radiale Säule und die proximale Ulna zusammen mit dem Koronoid haben eine entscheidende Bedeutung für den Erhalt der Gelenkstabilität und sollten daher bei der Revisionschirurgie bedacht werden. Bei schweren posttraumatischen degenerativen Veränderungen oder einer Gelenkdeformation kann unter Berücksichtigung des Patientenalters und etwaiger Kontraindikationen auch der sekundäre endoprothetische Ellenbogengelenkersatz in Erwägung gezogen werden.

Schlüsselwörter Instabilität · Komplikationen · Gelenkdeformation - Endoprothese $\cdot$ Koronoid

\section{Elbow fracture dislocations and Monteggia fractures. Posttraumatic corrections and revisions}

\section{Abstract}

In the case of revision or posttraumatic complications, further therapeutic options are often complex and require an individual approach due to the anatomical and biomechanical characteristics of the elbow joint. The radial column and the proximal ulna along with the coronoid process have a decisive influence on the preservation of joint stability and should therefore be carefully considered in revision surgery. Taking patient age and potential contraindications into consideration, secondary elbow arthroplasty can be an option if severe posttraumatic degenerative joint disease or joint deformities are present.

Keywords

Instability · Complications - Joint deformation . Prosthesis - Coronoid process ist in diesem Fall wie auch bei der Terrible-triad-Verletzung von Bedeutung. Ebenso ist die Wiederherstellung der Bandstabilität essenziell.

Die exakte anatomische Rekonstruktion des dorsalen Alignements der Ulna sowie die regelhafte Reposition im humeroradialen Gelenk sind bei der initialen operativen Therapie wichtig. Bei fehlerhafter Einstellung des dorsalen Alignements ist eine postoperative Achsabweichung des Unterarmes möglich, die zu funktionalen Einschränkungen führen kann. Nur eine sekundäre operative Achskorrektur kann in diesen Fällen die Beschwerden beheben.

Besteht zudem eine Fraktur des Koronoids, so ist das Fragment unter Berücksichtigung der Frakturmorphologie anatomisch zu refixieren, um zum einen die Gelenkstabilität zu erhalten und zum anderen die freie Umwendebewegung im proximalen Radioulnargelenk (PRUG) sicherzustellen. Die anatomische Rekonstruktion der Incisura trochlearis ist für die humeroulnare Artikulation ebenso bedeutsam. Bei Verlust des Koronoids besteht die Möglichkeit des endoprothetischen Ersatzes, wobei diese Therapieoption zum jetzigen Zeitpunkt noch weitestgehend experimentell ist. Wird der Radiuskopf endoprothetisch ersetzt, so kann alternativ das Koronoid mit einem knöchernen Radiuskopffragment augmentiert werden, um die anatomischen Verhältnisse wiederherzustellen.

\section{Komplexe Transolekranon- luxationsfraktur}

Nach schweren Transolekranonluxationsfrakturen ist trotz initialer anatomischer Gelenkrekonstruktion das Risiko für eine degenerative Gelenkde- 


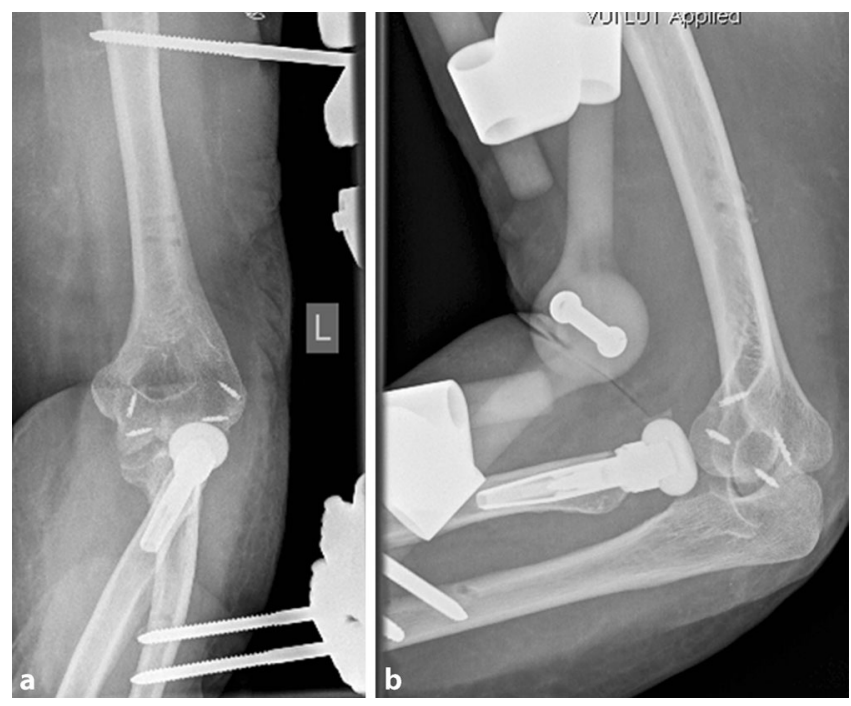

Abb. 3a,b $\Delta$ Postoperatives Röntgenbild nach erfolgreicher Revision bei Terrible-triad-Verletzung mit endoprothetischem Ersatz (MoPyC Radiuskopf-Prothese, Fa. Tornier) der radialen Säule, Refixation des lateralen und medialen Kollateralbandes mit humeral eingebrachten Fadenankern sowie Umsetzung der Schanz-Schrauben in Mini-open-Technik unter Retraktion der Weichteile und sicherem knöchernem Kontakt und Anbringung eines gelenküberschreitenden Bewegungsfixateurs.

(๑ Mit freundl. Genehmigung Prof. Dr. L. P. Müller, all rights reserved)
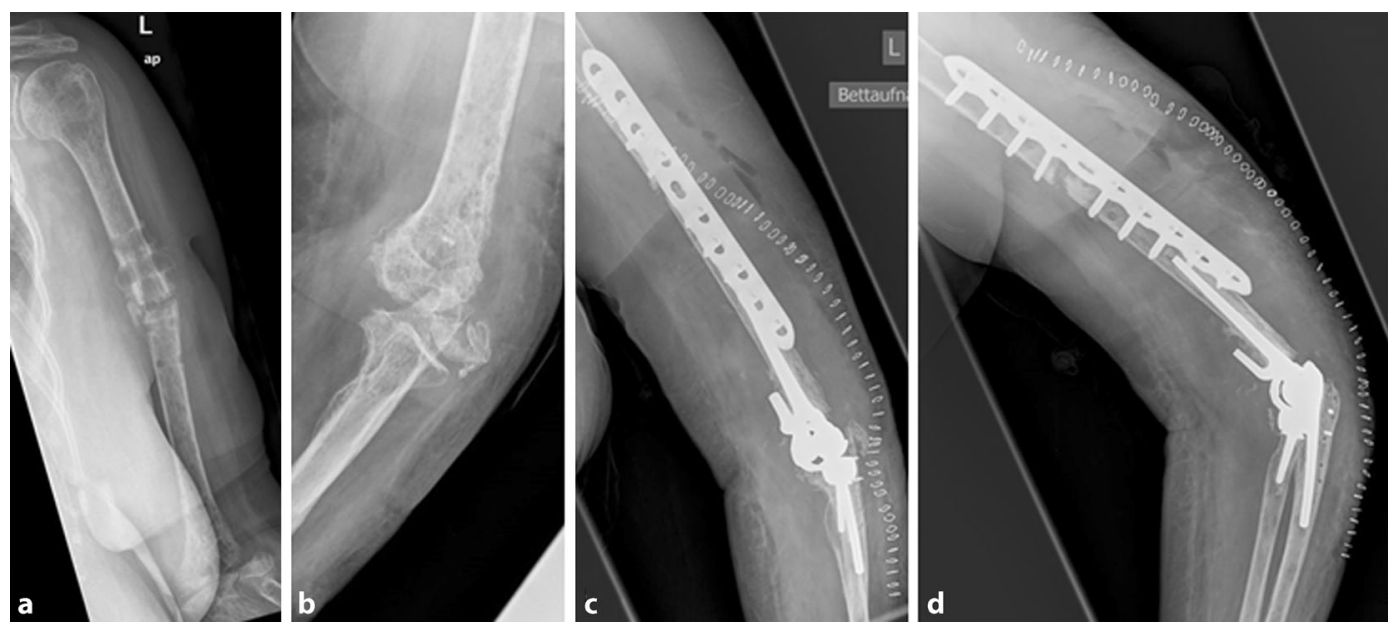

Abb. $4 \Delta$ a, b Nach stattgehabter offener Reposition und interner Fixierung einer Transolekranonluxationsfraktur mit zusätzlicher distaler Humerusfraktur zeigt sich im Verlauf eine schwere Kubitalarthrose mit begleitender Fraktur des Humerusschaftes im Bereich der ehemaligen Schanz-Schrauben nach Behandlung mittels Fixateur externe. c, $\mathbf{d}$ Unter Berücksichtigung des Patientenalters von 79 Jahren erfolgte neben der osteosynthetischen Versorgung der Humerusschaftfraktur der endoprothetische Ersatz des Ellenbogengelenkes mit einer zementierten, gekoppelten Latitude-Prothese (Fa. Tornier). (๑ Mit freundl. Genehmigung Prof. Dr. L. P. Müller, all rights reserved)

struktion erhöht. Das Risiko für andere Komplikationen wie beispielsweise eine persistierende Gelenkinstabilität oder eine sekundäre Frakturdislokation steigt ebenso an. Die anatomische Rekonstruktion der Ulna (Varusangulation, anteriore Deviation) sowie des Kapselbandapparates stehen im therapeutischen Fokus. Liegt begleitend eine distale Humerus- fraktur vor und kommt es hier zu einer sekundären Frakturdislokation oder einer Pseudarthrose des distalen Humerus nach initialer Osteosynthese, sollte zunächst stets die gelenkerhaltende Revisionsoperation angestrebt werden [20]. Durch eine Umpositionierung der distalen Humerusplatten und/oder durch einen Herstellerwechsel des verwendeten
Plattendesigns gelingt möglicherweise die suffiziente Re-Osteosynthese des distalen Humerus. Die Knochenheilung kann durch Anlagerung von z. B. autologem oder allogenem Knochenersatzmaterial unterstützt werden. Beim älteren Patienten (>75 Jahre) mit geringerem funktionalem Anspruch oder vorliegenden Komorbiditäten ermög- 
licht die zementierte und gekoppelte Ellenbogentotalendoprothese (EBTEP) eine umgehende stabile Gelenkversorgung und Schmerzreduktion, wodurch eine frühfunktionale Nachbehandlung ermöglicht wird (•Abb. 4a-d). Die Indikation zum endoprothetischen Ersatz muss individuell erfolgen und kann möglicherweise auch schon bei Patienten unter 75 Jahren gestellt werden (z. B. bei geringem funktionalem Anspruch, schwerer Grunderkrankung mit reduzierter Lebenserwartung etc.). Unter Berücksichtigung der notwendigen Gewichtslimitation $(<5 \mathrm{~kg})$ nach EBTEP lassen sich bei sichergestellter Patientencompliance gute bis sehr gute funktionelle Ergebnisse erzielen [21, 22].

\section{Fazit für die Praxis}

\section{- Komplexe Ellenbogenverletzungen benötigen bereits initial komplexe Therapieregimes, um die Wiederher- stellung der anatomischen osteoli- gamentären Gelenkverhältnisse zu ermöglichen.}

- Anamnese und weiterführende Bildgebung sind zur Revisionsplanung wichtig.

- Revisionen nach komplexen Ellenbogenverletzungen sind oftmals kompliziert und erfordern zum Teil individuelle Entscheidungen.

- Im Revisionsfall gilt es - unter Berücksichtigung der anatomischen Strukturen - das Gelenk zu stabilisieren. Der Erhalt der radialen Säule ist anzustreben.

- Koronoidfragmente sollten unter Berücksichtigung der Frakturmorphologie zum Erhalt der Stabilität refixiert werden. Die anatomische Koronoidreposition ist für die freie Bewegung im PRUG und im Humeroulnargelenk entscheidend.

- Bei fortgeschrittener Kubitalarthrose ist der endoprothetische Gelenkersatz beim älteren Patienten (>75 Jahren) eine Möglichkeit zur umgehenden Gelenkstabilisation mit Schmerzreduktion und frühfunktionaler Nachbehandlung.

\section{Korrespondenzadresse

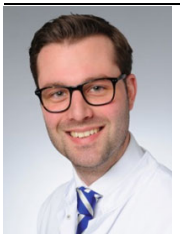 \\ Dr. med. C. Ries \\ Klinik und Poliklinik für Or- thopädie und Unfallchirurgie, Universitätsklinik Köln Kerpener Str. 62, 50937 Köln, Deutschland christian.ries@uk-koeln.de}

\section{Einhaltung ethischer Richtlinien}

Interessenkonflikt. Das Zentrum für Biomechanik der Klinik und Poliklinik für Orthopädie und Unfallchirurgie derUniversitätzu Köln wird mit einem jährlichen Betrag der Firmen Synthes und Medartis unterstützt aber nicht im Speziellen für die vorliegende Arbeit. K. Wegmann und L.P. Müller sind als Berater für Tornier und Medartis tätig. K. Wegmann und L.P. Müller haben und werden keinen finanziellen Betrag in irgendeiner Form erhalten. Kein Betrag wurde oder wird direkt oder indirekt in Zusammenhang mit dem vorliegenden Artikel gezahlt. C. Ries, M. Hackl und T. Leschinger geben an, dass kein Interessenskonflikt besteht.

Dieser Beitrag beinhaltet keine von den Autoren durchgeführten Studien an Menschen oder Tieren.

The supplement containing this article is not sponsored by industry.

\section{Literatur}

1. Ring D, Jupiter JB, Zilberfarb J (2002) Posterior dislocation of the elbow with fractures of the radial head and coronoid. J Bone Joint Surg Am 84:547-551 (PubMed PMID: 11940613)

2. Kälicke T, Muhr G, Frangen TM (2007) Dislocation of the elbow with fractures of the coronoid process and radial head. Arch Orthop Trauma Surg 127(10):925-931 (Epub 2007 Aug 23. PubMed PMID: 17713772)

3. Giannicola G, Calella P, Piccioli A et al (2015) Terrible triad of the elbow: is it still a troublesome injury? Injury 46(Suppl 8):S68-S76 (PubMed PMID: 26747922). doi:10.1016/S0020-1383(15)30058-9

4. Lindenhovius AL, Jupiter JB, Ring D (2008) Comparison of acute versus subacute treatment of terrible triad injuries of the elbow. J Hand Surg Am 33(6):920-926 (PubMed PMID: 18656766). doi:10.1016/j.jhsa.2008.02.007

5. Fitzgibbons PG, Louie D, Dyer GS et al (2014) Functional outcomes after fixation of "terrible triad" elbow fracture dislocations. Orthopedics 37(4):373-376 (PubMed PMID: 24762843). doi:10.3928/01477447-20140401-59

6. Chen HW, Liu GD, Wu LJ (2014) Complications of treating terrible triad injury of the elbow: a systematic review. PLOS One 9(5):e97476 (eCollection 2014. Review. PubMed PMID: 24832627; PubMed Central PMCID: PMC4022574). doi:10.1371/ journal.pone.0097476

7. Morrey BF, An KN, Stormont TJ (1988) Force transmission through the radial head. J Bone Joint Surg Am 70:250-256

8. Johnson JA, Beingessner DM, Gordon KD (2005) Kinematics and stability of the fractured and implant-reconstructed radial head. J Shoulder Elbow Surg 14(1 Suppl S):S195-S201 (Review. PubMed PMID: 15726082)

9. Doornberg JN, Linzel DS, Zurakowski D et al (2006) Reference points for radial head prosthesis size. J Hand Surg Am 31(1):53-57 (PubMed PMID: 16443104)

10. Hartzler RU, Llusa-Perez M, Steinmann SP et al (2014) Transverse coronoid fracture: When does it have to be fixed? Clin Orthop Relat Res 472(7):2068-2074 (PubMed PMID: 24585321; PubMed Central PMCID: PMC4048411). doi:10.1007/s11999-014-3477-1

11. O'Driscoll SW, Jupiter JB, Cohen MSR et al (2003) Difficult elbow fractures: pearls and pitfalls. Instr Course Lect 52:113-134

12. McGinley JC, Hopgood BC, Gaughan JP et al (2003) Forearm and elbow injury: the influence of rotational position. J Bone Joint Surg Am 85:2403-2409

13. Burkhart KJ, Franke S, Wegmann K et al (2015) Mason I fracture - a simple injury? Unfallchirurg 118(1):9-17 (Review. German. PubMed PMID: 24452244). doi:10.1007/s00113-013-2532-9

14. McGinley JC, Gold G, Cheung E et al (2014) MRI detection of forearm soft tissue injuries with radial head fractures. Hand 9(1):87-92 (PubMed PMID: 24570643; PubMed Central PMCID: PMC3928382). doi:10.1007/s11552-013-9561-2

15. Shepard MF, Markolf KL, Dunbar AM (2001) Effects of radial head excision and distal radial shortening on load-sharing in cadaver forearms. J Bone Joint Surg Am 83-A(1):92-100

16. Goldberg I, Peylan J, Yosipovitch Z (1986) Late results of excision of the radial head for an isolated closed fracture. J Bone Joint Surg Am 68(5):675-679

17. Monteggia GB (1814) Istruzioni chirurgiche Bd. 5 . Pirotta \& Maspero, Milano, S52-138

18. Llusà Perez M, Lamas C, Martínez I et al (2002) Monteggia fractures in adults. Review of 54 cases. Chir Main 21(5):293-297 (PubMed PMID: 12491706)

19. Strauss EJ, Tejwani NC, Preston CF et al (2006) The posterior Monteggia lesion with associated ulnohumeral instability. J Bone Joint Surg $\mathrm{Br}$ 88(1):84-89 (PubMed PMID: 16365126)

20. Cil A, Veillette CJ, Sanchez-Sotelo J et al (2008) Linked elbow replacement: a salvage procedure for distal humeral nonunion. J Bone Joint Surg Am 90:1939-1950

21. Müller LP, Wegmann K, Burkhart KJ (2013) Frakturendoprothetik der distalen Humerusfraktur. Unfallchirurg 116(8):708-715. doi:10.1007/s00113013-2411-4

22. Müller LP, KamineniS, RommensPM(2005) Primary total elbow replacement for fractures of the distal humerus. Oper Orthop Traumatol 17(2):119-142 